\title{
On the number of sums and products
}

\author{
by \\ GyörGY ELEKES (Budapest) \\ Dedicated to the memory of P. Erdös
}

In what follows $\mathcal{A}$ will always denote a finite subset of the non-zero reals, and $n$ the number of its elements. As usual, $\mathcal{A}+\mathcal{A}$ and $\mathcal{A} \cdot \mathcal{A}$ stand for the sets of all pairwise sums $\left\{a+a^{\prime}: a, a^{\prime} \in \mathcal{A}\right\}$ and products $\left\{a \cdot a^{\prime}: a, a^{\prime} \in \mathcal{A}\right\}$, respectively. Also, $|S|$ denotes the size of a set $S$.

The following problem was posed by Erdős and Szemerédi (see [5]):

For a given $n$, how small can one make $|\mathcal{A}+\mathcal{A}|$ and $|\mathcal{A} \cdot \mathcal{A}|$ simultaneously? In other words, defining

$$
m(\mathcal{A}):=\max \{|\mathcal{A}+\mathcal{A}|,|\mathcal{A} \cdot \mathcal{A}|\},
$$

a lower estimate should be found for

$$
g(n):=\min _{|\mathcal{A}|=n} m(\mathcal{A}) .
$$

Rem ark. The philosophy behind the question is that either of $|\mathcal{A}+\mathcal{A}|$ or $|\mathcal{A} \cdot \mathcal{A}|$ is easy to minimize - just take an arithmetic or geometric (i.e., exponential) progression for $\mathcal{A}$. However, in both of these examples, the other set becomes very large.

In their above mentioned paper, Erdős and Szemerédi managed to prove the existence of a small but positive constant $c_{1}$ such that $g(n) \geq n^{1+c_{1}}$ for all $n$. (See also p. 107 of Erdős' paper [3].) Later on, Nathanson and K. Ford found the lower bounds $n^{32 / 31}$ and $n^{16 / 15}$, respectively [7].

The goal of this paper is to improve the exponent to $5 / 4$.

THEOREM 1. There is a positive absolute constant $c$ such that, for every $n$-element set $\mathcal{A}$,

$$
c \cdot n^{5 / 4} \leq \max \{|\mathcal{A}+\mathcal{A}|,|\mathcal{A} \cdot \mathcal{A}|\} .
$$

1991 Mathematics Subject Classification: Primary 05D99, 11B75, 52C10; Secondary 11B25, 51A99, 51H10.

Partially supported by HU-NSF grants OTKA T014105, T014302 and T019367. 
1. A tool from geometry. In the proof we shall use a result of Szemerédi and Trotter (see [9]).

Proposition 1 (Szemerédi-Trotter Theorem). Let $t$ and $N$ be positive integers with $t^{2} \leq N$. Let, moreover, $\mathcal{P}$ be a set of $N$ distinct points in the plane and $e_{1}, \ldots, e_{M}$ some (also distinct) straight lines. If each of the $e_{i}$ contains $t$ or more points of $\mathcal{P}$, then

$$
M \leq C \cdot \frac{N^{2}}{t^{3}}
$$

(Here $C$ is a huge absolute constant of Szemerédi and Trotter-improved later to the more reasonable value of 3 by Clarkson et al. [2]. Recently, a simple and very elegant proof was found by Székely [8].)

Remark. The importance of the above assertion lies in the fact that the exponent of $t$ in the denominator is strictly larger than 2. (A bound of $M \leq\left(\begin{array}{c}N \\ 2\end{array}\right) /\left(\begin{array}{c}t \\ 2\end{array}\right)$ would be trivial by just double-counting the pairs of points.) The first result in this direction was that of Beck [1], with an exponent 2.05 of $t$; this was later improved to $t^{3}$ by Szemerédi and Trotter.

2. Proof of the Theorem. Denote the elements of $\mathcal{A}$ by $a_{1}, \ldots, a_{n}$, and define the following $n^{2}$ functions:

$$
f_{j, k}(x):=a_{j}\left(x-a_{k}\right) \quad \text { for } 1 \leq j, k \leq n .
$$

LEMma 2. For every $j, k \leq n$, the function $f_{j, k}$ maps at least $n$ elements of $\mathcal{A}+\mathcal{A}$ to some elements of $\mathcal{A} \cdot \mathcal{A}$.

(Indeed, the image of $a_{k}+a_{i}$ is $a_{j} \cdot a_{i} \in \mathcal{A} \cdot \mathcal{A}$, for every $a_{i} \in \mathcal{A}$.)

From a geometric point of view, the above lemma asserts that the graph of each of the functions $f_{j, k}$ contains $n$ or more points of $\mathcal{P}:=(\mathcal{A}+\mathcal{A}) \times$ $(\mathcal{A} \cdot \mathcal{A})$. Put $N=|\mathcal{P}|=|(\mathcal{A}+\mathcal{A})| \cdot|(\mathcal{A} \cdot \mathcal{A})|$. Then, by applying Proposition 1 to $\mathcal{P}$ and the $f_{j, k}$ (with $M=n^{2}$ and $t=n$ ), we get

$$
n^{2} \leq C \cdot \frac{N^{2}}{n^{3}}
$$

i.e., $N \geq C^{-1 / 2} n^{5 / 2}$-whence the Theorem follows immediately.

\section{References}

[1] J. Beck, On the lattice property of the plane and some problems of Dirac, Motzkin and Erdös, Combinatorica 3 (1983), 281-297.

[2] K. Clarkson, H. Edelsbrunner, L. Guibas, M. Sharir and E. Welzl, Combinatorial complexity bounds for arrangements of curves and surfaces, Discrete Comput. Geom. 5 (1990), 99-106.

[3] P. Erdős, On some of my favorite theorems, pages 97-132 of [6]. 
[4] P. Erdős, L. Alpár and G. Halász (eds.), Studies in Pure Mathematics, To the Memory of Paul Turán, Akadémiai Kiadó-Birkhäuser, Budapest-Basel, 1983.

[5] P. Erdős and E. Szemerédi, On sums and products of integers, pages 213-218 of $[4]$.

[6] D. Miklós, V. T. Sós and T. Szőnyi (eds.), Combinatorics, Paul Erdős is Eighty, János Bolyai Math. Soc., Budapest, 1996.

[7] M. B. Nathanson, personal communication.

[8] L. A. Székely, Crossing numbers and hard Erdös problems in Discrete Geometry, Combin. Probab. Comput., 1997, to appear.

[9] E. Szemerédi and W. T. Trotter, Jr., Extremal problems in discrete geometry, Combinatorica 3 (1983), 381-392.

Department of Computer Science

Eötvös University

Múzeum krt. 6-8

H-1088 Budapest, Hungary

E-mail: elekes@cs.elte.hu 